\title{
Diferenciación o paralelismo entre hermanos, ¿Influye la relación con
}

\author{
los padres? \\ Differential or parallel influences, Do relationships with parents \\ matter?
}

David Posada, Silvia López Larrosa

Universidade da Coruña,

\begin{abstract}
Resumen
Este estudio analiza la relación entre tres dimensiones parentales: afecto, control y justicia, y la diferenciación y el paralelismo de 41 parejas de hermanos adolescentes. Los datos indicaron que cuanto más justo es el trato de los progenitores hacia los hijos percibido por los hermanos menores, más altas son las puntuaciones en paralelismo y más bajas en diferenciación. Cuanto más controlan los padres, según el hijo mayor, más bajas son las puntuaciones en paralelismo. Estos datos muestran que el estilo parental se relaciona con los procesos de diferenciación y paralelismo en hermanos.

Palabras clave: hermanos, influencia paralela, influencia diferencial, padres
\end{abstract}

\begin{abstract}
This study analyzes the relationships between three parental behaviors: affect, control and justice, and the differential and parallel influences of 41 adolescent sibling dyads. When younger siblings perceived fairness increases, so do the scores in parallelism while scores in differentiation decrease. When parents control more, according to the older sibling, the scores in parallelism are lower. These data show that the parental style is related to siblings' processes of differentiation and parallelism.

Keywords: siblings, parallel influences, differential influences, parents.
\end{abstract}

\section{Introducción}

El proceso de diferenciación se puede definir como la búsqueda que los hijos realizan de su propia identidad y singularidad dentro de la familia. Es un proceso mediante el cual los hijos tratan de distinguirse de sus hermanos y hermanas desarrollando diferentes cualidades e intereses en un esfuerzo por evitar la competencia directa por los recursos y establecer su propio papel e identidad dentro de la familia (Sulloway, 1996; Branje, Van Lieshout, Van Aken y Haselager, 2004). Este proceso, que parece fruto de la decisión consciente o inconsciente de los hijos, también se ve influido por las propias diferencias entre los hermanos, ya que el "ambiente compartido" puede afectar de forma distinta a cada uno de ellos (Turkheimer y Waldron, 2000).

El proceso de diferenciación puede intensificarse durante la adolescencia, cuando los hijos buscan una mayor autonomía de la familia y se agudiza la búsqueda y el desarrollo de su identidad. Esta diferenciación no presupone conflicto entre los hermanos sino que, durante el proceso de diferenciación, los hermanos pueden mantener buenas relaciones entre ellos (Feinberg, McHale, Crouter, y Cumsille, 2003).

Por su parte, la influencia paralela hace referencia al papel que un hermano juega como modelo y fuente de aprendizaje del otro al permitirle el acceso a diversos contextos o compañías (Whiteman, McHale y Crouter, 2007).

Dado que el comportamiento de los padres, su estilo educativo, es una de las formas más poderosas de influir el comportamiento de los hijos (Biglan, Flay, Embry y Sandler, 2012), en esta investigación nos centraremos en analizar el papel que juegan las conductas de los padres en el deseo de los adolescentes de parecerse o diferenciarse de sus hermanos. En las conductas parentales consideraremos el control, el afecto y la justicia del trato de los padres percibidos por los hijos. El control se entiende en este estudio en el sentido de supervisión. Por su parte, el afecto hace referencia a aceptación/implicación o calidez (Antón, Seguí, Antón y Barrera, 2016). Estas dos dimensiones, pese al empeño de los padres, no suelen manifestarse por igual hacia todos los hijos (Plomin, Asbury, Dunn, 2001). Sin embargo, pocos padres, aproximadamente el $40 \%$, reconocen que estas diferencias se producen (Daniels et al., 1985). La clave según las investigaciones está en si, pese a las diferencias, los hermanos perciben que el trato es justo. Si los hijos perciben un trato diferente, o incluso muy diferente, pero justo, las relaciones entre hermanos son más positivas. $\mathrm{Si}$ las consideran injustas, esto suele conllevar una peor relación entre los hermanos (Kowall, Kramer, Krull y Crick, 2002). 
Por esto, el objetivo de este estudio es analizar la relación entre las conductas de los padres (control, afecto y justicia) y los procesos de diferenciación o paralelismo entre los hermanos.

\section{Método}

\section{Participantes}

En este estudio participaron alumnos de Educación Secundaria de tres centros educativos de las provincias de A Coruña y Pontevedra. Dos centros eran públicos y uno era concertado. En total se contó con 82 participantes, 44 chicos y 38 chicas, repartidos en 41 parejas de hermanos con edades comprendidas entre los 12 y los 18 años. La media de edad de los hermanos mayores era de 15.49 años $(D E=1.14)$ y era de 12.98 años $(D E=1.15)$ en el caso de los hermanos menores. En 26 parejas los hermanos tenían sexos diferentes, mientras que en 9 las parejas eran de chicas y en 6 eran solo de chicos.

\section{Instrumentos}

SIS (Sibling Influence Scale) de Whiteman, Bernard y McHale (2010). Este cuestionario consta de 18 preguntas repartidas en dos subescalas. La escala de influencia paralela consta de 8 ítems, por ejemplo "Mi hermana/o aporta un modelo de como yo debería actuar". Mientras que la escala de diferenciación consta de 10 ítems, por ejemplo "Trato de ser diferente a mi hermana/o". La fiabilidad de la escala de influencia paralela para el hermano mayor es de $\alpha=.84$ y para el hermano menor es de $\alpha=.70$. Por otro lado, la fiabilidad de la escala de diferenciación es de $\alpha=.75$ para el hermano mayor y es de $\alpha=.62$ para el hermano menor. Para este estudio agrupamos los datos de ambos hermanos, de modo que obtuvimos una única puntuación de diferenciación con la suma de las escalas de los hermanos mayores y menores, y una única puntuación de paralelismo con la suma de las puntuaciones de los hermanos mayores y menores. La fiabilidad de la escala de paralelismo así calculada es de $\alpha=.79$. La fiabilidad de la escala de diferenciación es de $\alpha=.73$.

SIDE (Sibling Inventory of Differential Experience) de Daniels y Plomin (1985). Es un cuestionario de 73 ítems que explora las interacciones de los hermanos de una díada y el comportamiento de los padres hacia ellos. Para este estudio, se seleccionaron los cinco ítems de la subescala de afecto y los 4 ítems de la subescala de control. En ambos casos se recoge información del padre y la madre con respecto a cada pareja de hermanos tal y como los percibe cada hijo. Para contestar, se usa una escala tipo Likert de cinco puntos con opciones de respuesta que van de 1 "Hacia mi hermano/a mucho más" hasta 5 "Hacia mi mucho más".

En este estudio, la fiabilidad del afecto materno hacia el hermano mayor es de $\alpha=.77 \mathrm{y}$ es de $\alpha=.71$ en el caso del hermano menor. La escala de afecto paterno hacia el hermano mayor es de $\alpha=.65$ y es de $\alpha=.73$ para el hermano menor. Por su parte, la fiabilidad de la escala de control materno hacia el hermano mayor es de $\alpha=.46$ y es de $\alpha=.62$ para el hermano menor. La fiabilidad de la subescala de control paterno hacia el hermano mayor es de $\alpha=.65$ y es de $\alpha=.56$ para el hermano menor.

Para evaluar la justicia, se desarrollaron cuatro preguntas que exploran la justicia del trato del padre y la madre hacia cada uno de los hijos percibida por el hermano mayor y el hermano menor. Contestan usando una escala tipo Likert de 5 puntos con opciones de respuesta que van de 1 a 5 siendo el 1 "nada justo" y el 5 "muy justo". La fiabilidad de la medida de justicia percibida por el hermano mayor con respecto a sí mismo y su hermano es de $\alpha=.90$. La fiabilidad de la medida de justicia percibida por el hermano menor hacia sí mismo y su hermano mayor es de $\alpha=.86$.

Además de estos instrumentos y preguntas, se recogen datos sociodemográficos como el sexo de los hermanos, la edad y el lugar de residencia.

\section{Procedimiento}

Se contactó con los centros educativos y se pidió permiso a sus directores. Desde el centro identificaron a las parejas de hermanos que cumplían los criterios de inclusión: hermanos adolescentes, estudiantes de Secundaria y sin discapacidad. A estas parejas de hermanos se les entregó una carta informativa que debían dar a sus padres. En la carta se presentaba el estudio y se solicitaba su permiso para que sus hijos participaran en el estudio. Aquellos hermanos que contaban con el permiso de sus padres, se reunieron en un aula del centro escolar y contestaron sus cuestionarios individualmente. Un investigador estuvo en la sala en todo momento por si surgían dudas durante la cumplimentación del cuestionario. Este mismo investigador dio todas las informaciones iniciales y les garantizó que sus respuestas eran anónimas. Para ello, se asignó a cada díada un número de identificación.

\section{Resultados}

Para responder a nuestro objetivo, primeramente se sumaron las puntuaciones de afecto y control del padre y la madre hacia cada hijo, de modo que se obtuvo una puntuación de afecto (suma de padre y madre) y otra de control (suma de padre y madre) para cada hijo. Se realizaron análisis de correlaciones entre las puntuaciones de diferenciación e influencia paralela de los hermanos. Los datos indicaron que no hay relaciones significativas $(p>.10)$ entre los procesos de diferenciación y paralelismo de los hermanos mayores por un lado y de los hermanos menores por otro; ni al considerar las escalas de diferenciación y paralelismo sumando los valores de hermanos mayores y menores en cada escala $(p>.10)$

A continuación, se realizaron análisis de regresión lineal múltiple por pasos tomando la justicia, el afecto y el control como variables independientes, y considerando como variables dependientes la influencia paralela y la diferenciación de los hermanos.

$\mathrm{Al}$ analizar los datos de los procesos de influencia paralela de los hermanos, se extrajo un modelo de dos factores, en el que el $13 \%$ de la influencia paralela la 
explica el control de padre y madre percibida por el hijo mayor, mientras que esta variable junto con la justicia del trato de padres y madres que percibe el hijo menor explican el $23 \%$ de la varianza. Cuanto más control percibe el hijo mayor en el trato de sus padres, más baja es la puntuación en influencia paralela mientras que cuando más elevada es la justicia del trato de los padres percibida por el hijo menor, más alta es la puntuación en influencia paralela (tabla 1).

Tabla1

Resultados del análisis de regresión de influencia paralela percibida por los hermanos tomando como variables independientes el afecto, el control y la justicia percibida del trato de padres y madres.

\begin{tabular}{lcccc}
\hline Modelo & $\begin{array}{c}R^{2} \\
\text { ajustado }\end{array}$ & $\begin{array}{c}\text { Cambio } \\
\text { en } R^{2}\end{array}$ & $\begin{array}{c}\text { Cambio } \\
\text { en } F\end{array}$ & Significación \\
\hline 1 & 0.13 & 0.16 & 5.66 & 0.04 \\
2 & 0.23 & 0.12 & 4.73 & 0.03 \\
\hline \multicolumn{5}{c}{ Influencia } \\
Paralela & $\begin{array}{c}\text { Control de } \\
\text { padre/madre } \\
\text { hacia hijo } \\
\text { mayor }\end{array}$ & $\begin{array}{c}\text { Justicia } \\
\text { percibida } \\
\text { por hijo } \\
\text { menor hacia } \\
\text { él y su } \\
\text { hermano }\end{array}$ \\
\hline Influencia & & & & \\
Paralela & & & & \\
$\beta$ & & & \\
Error & & & & \\
Estándar & & & 0.36 & $(0.18)$ \\
\end{tabular}

Al considerar la diferenciación, nuevamente la justicia percibida por el hijo menor fue la variable significativa, aunque en este caso explica un menor porcentaje de la varianza, el $12 \%$. Cuanto más justo es el trato de los padres hacia los hermanos percibido por los hijos pequeños, menos tratan de diferenciarse los hermanos entre sí (tabla 2).

Tabla 2

Resultados del análisis de regresión de los procesos de diferenciación de los hermanos tomando como variables independientes el afecto, el control y la justicia percibida del trato de padres y madres.

\begin{tabular}{|c|c|c|c|c|}
\hline Modelo & $\begin{array}{c}R^{2} \\
\text { ajustado }\end{array}$ & $\begin{array}{c}\text { Cambio } \\
\text { en } R^{2}\end{array}$ & $\begin{array}{c}\text { Cambio } \\
\text { en } F\end{array}$ & Significación \\
\hline \multirow[t]{2}{*}{1} & 0.12 & 0.15 & 5.34 & 0.02 \\
\hline & & \multicolumn{2}{|c|}{$\begin{array}{c}\text { Influencia } \\
\text { Paralela }\end{array}$} & $\begin{array}{c}\text { Justicia } \\
\text { percibida por } \\
\text { hijo menor } \\
\text { hacia él y su } \\
\text { hermano }\end{array}$ \\
\hline \multicolumn{5}{|c|}{ Diferenciación } \\
\hline \multicolumn{2}{|l|}{$\beta$} & \multicolumn{3}{|r|}{$\begin{array}{l}-.38 \\
(.04)\end{array}$} \\
\hline
\end{tabular}

\section{Discusión}

Los datos indican que la justicia del trato de los padres parece la variable más relevante de las tres analizadas a la hora de explicar los procesos de diferenciación y paralelismo de los hermanos. Nuestros resultados muestran que los procesos de diferenciación y paralelismo no son complementarios, esto es, uno no es la imagen "especular" del otro, aunque sí se mantiene la coherencia. Así, la justicia funciona en un sentido al considerar el paralelismo y en el sentido opuesto al considerar la diferenciación. Por ello, cuanto más justo es el trato que percibe el hijo menor, más paralelismo y menos diferenciación se da en los hermanos. No obstante, el control también es una variable relevante en nuestro estudio con respecto al paralelismo. Así, cuanto más control hacia el hermano mayor, menos paralelismo se da en los hermanos.

Estos resultados destacan la importancia que tiene el trato de los padres hacia los hijos. Muestran que según sea este trato de controlador o de justo influirá en el deseo de parecerse a, o por el contrario diferenciarse de, un hermano. Este estudio es una aportación a un ámbito todavía poco explorado. A partir de aquí, sería interesante estudiar la influencia que pueden tener los padres en las diferencias entre los hermanos en diversos ámbitos del desarrollo adolescente.

\section{Referencias bibliográficas}

Antón, J. M., Seguí, D., Antón, L. y Barrera, A. (2016). Relación entre estilos parentales, intensidad psicopatológica y tipo de sintomatología en una muestra clínica adolescente. Anales de Psicología, 32 ,

417-423.

http://dx.doi.org/10.6018/analesps.32.2.203871

Biglan, A., Flay, B. R., Embry, D. D., y Sandler, I. N. (2012). The critical role of nurturing environments for promoting human well-being. American Psychologist, 67, 257-271. http://dx.doi.org/10.1037/a0026796

Branje, S., Van Lieshout, C., Van Aken, M., y Haselager, G. (2004). Perceived support in sibling relationships and adolescent adjustment. Journal of Child Psychology and Psychiatry, 45, 1385-1396. http://dx.doi.org/10.1111/j.1469-7610.2004.00332.x

Daniels, D., Dunn, J., Furstenberg, F. F. y Plomin, R. (1985). Environmental differences within the family and adjustment differences within pair of adolescent siblings. Child Development, 56, 764-774. http://dx.doi.org/10.2307/1129765

Daniels, D. y Plomin, R. (1985). Differential experience of siblings in the same family. Developmental Psychology, 23, 747-760. http://dx.doi.org/10.1037/0012-1649.21.5.747

Feinberg, M. E., McHale, S. M., Crouter, A. C., \& Cumsille, P. (2003). Sibling differentiation: Sibling and parent relationship trajectories in adolescence. Child Development, 74, 1261-1274. http://dx.doi.org/110.1111=1467-8624.00606

Kowal, A., Kramer, L., Krull, J., Crick, N. (2002). Children's perceptions of the fairness of parental preferential treatment and their socioemotional well- 
being. Journal of Family Psychology, 16, 297306. http://dx.doi.org/10.1037/0893-3200.16.3.297

Kowal, A.K., Krull, J.L. y Kramer, L. (2004). How the differential treatment of siblings is linked with parent-child relationship quality. Journal of Family Psychology, 18 ,

658 -

665. http://dx.doi.org/10.1037/0893-3200.18.4.658

Kowal, A. K., Krull, J. L., \& Kramer, L. (2006). Shared understanding of parental differential treatment in families. Social Development, 15, 276$295 . \quad$ http://dx.doi.org/10.1046/j.14679507.2006.00341.x

Plomin, R., Asbury, K., Dunn, J. (2001). Why are children in the same family so different? Non-shared environment a decade later. Canadian Journal of Psychiatry, 46 , 225-233. https://doi.org/10.1177/070674370104600302

Sulloway, F.J. (1996). Born to rebel: Birth order, family dynamics, and creative lives. New York, NY: Pantheon Books.

Turkheimer, E. y Waldron, M. (2000). Nonshared environment: a theoretical, methodological, and quantitative review. Psychological Bulletin, 126, 78108. http://dx.doi.org/10.1037/0033-2909.126.1.78

Whiteman, S. D., Bernard, J. M., \& McHale, S. M. (2010). The nature and correlates of sibling influence in two-parent African American families. Journal of Marriage and Family, 72, 267-281. http://dx.doi.org/10.1111/j.1741-3737.2010.00698.x

Whiteman, S.D., McHale, S. M., \& Crouter, A. C. (2007). Explaining sibling similarities: Perceptions of sibling influences. Journal of Youth and Adolescence, 36, 963-972. http://dx.doi.org/ 10.1007/s10964-006-9135 